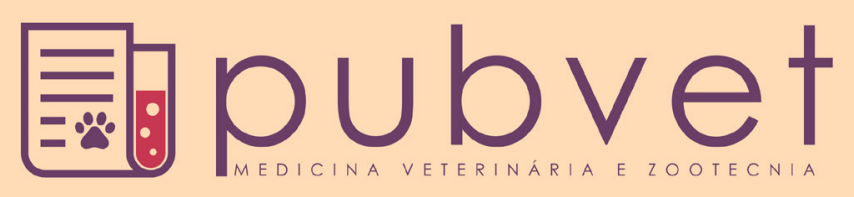

HTTP://DX.DOI.ORG/10.22256/PUBVET.V11N5.452-459

\title{
Resíduos de agrotóxicos do grupo de ditiocarbamatos em maçãs (Malus domestica Borkh.) in natura no Brasil - 10 anos
}

\author{
Cristina Link Runtzel ${ }^{1 *}$, Simone Galvão², Marcella Nunes Pereira ${ }^{2}$, Carlos Eduardo \\ Soares $^{2}$, Vildes Maria Scussel ${ }^{3}$
}

${ }^{1}$ Graduanda, ${ }^{2}$ Pós-graduandos e ${ }^{3}$ Professora Doutora em Ciências de Alimentos, Laboratório de Micotoxicologia e Contaminantes Alimentares, Departamento de Ciência e Tecnologia de Alimentos, Centro de Ciências Agrárias, Universidade Federal de Santa Catarina, Florianópolis, SC, Brasil.

Autor para correspondência, E-mail: link.cristina12@gmail.com

RESUMO. Realizou-se uma análise crítica dos dados obtidos do Programa de Análise de Resíduos de Agrotóxicos (PARA) da Agência Nacional de Vigilância Sanitária (ANVISA) sobre os resíduos de agrotóxicos do grupo químico ditiocarbamato (DTCs) em maçãs (Malus domestica Borkh) comercializadas in natura durante dez anos no pais. Foram analisadas 1422 amostras de maçãs, das quais 605 apresentaram algum nível de contaminação. O número de amostras positivas para DTCs foi de somente $32.1 \% \mathrm{em} 2003$, já o ano seguinte (2004) configurou como o período de maior número de amostras contaminadas, correspondendo a 83,9\%, seguido pelo ano de 2007 com $81,9 \%$. As maçãs atingiram níveis de até $3,04 \mathrm{mg} / \mathrm{Kg}$ no ano de 2005 , excedendo o limite máximo de resíduos (LMR) (2 mg/Kg) nacional. Independente dos dados informados, o Programa não informa quantas e/ou a porcentagem de amostras que excedeu o LMR, somente a faixa. Os valores encontrados foram satisfatórios ( $\leq$ LMR) nos anos de 2003, 2006 e 2007. Foi possível perceber que as maçãs brasileiras, ao longo do período de avaliação oscilaram em picos de presença de resíduo acima dos LMR (em 2001/2002, 2005, 2009, 2010 e 2012), expondo consumidores aos efeitos adversos dos DTCs. Considerando a faixa de anos do monitoramento realizado pelo PARA e avaliada no presente estudo, foi observado que não houve evolução nos resíduos detectados (redução), muito menos acréscimo no número de amostras analisado (oscilou em todos os períodos), bem como de laboratório oficiais para análise, o que demonstra a necessidade de aprimoramento desses fatores. Há necessidade da ANVISA continuar a informar oficialmente consumidores (via website) já que este processo informativo parou em 2012.

Palavras chave: consumidores, ditiocarbamatos, maçãs, resíduos, PARA/ANVISA

\section{Dithiocarbamates pesticide residues evolutionin fresh apples (Malus domestica Borkh.) in Brazil - ten years}

ABSTRACT. A critical analysis of the dithiocarbamate (DTCs) residues (ten years) in fresh apples (Malus domestica Borkh.) reported by the Pesticide Residue Analysis Program (PARA) of the Brazilian Sanitary Surveillance Agency (ANVISA) was carried out. From the total of 1422 apples samples, 605 of them present some levels of contamination. Although the number of positive samples for DTCs were rather low (32.1\%) in 2003, the following year (2004) had the highest number of contaminated apples samples (135) corresponding to 83.9\%, followed by 2007 with 81.9\% (113). Apples in Brazil reached residue levels up to $3.04 \mathrm{mg} / \mathrm{kg}$ in 2005, exceeding the country MRL ( $2 \mathrm{mg} / \mathrm{kg}$ ). Despite that, the Program does not inform how many and/or the percentage that effectively exceeded that limit. The values were satisfactory $(\leq \mathrm{MLR})$ in the prior to 2003 years and so 
in 2006 and 2007. According to the data analyzed, the apples commercialized in Brazil over the evaluation period, had residue above the MRLs level in bouncing years (2001/2002, 2005, 2009, 2010 and 2012), exposing consumers to the adverse DTCs effects. Considering the ten years evaluated, it was observed that there were no evolution on the residues detected (reduction), nor in the number of samples surveyed or official laboratories for analysis (increase), thus those factors should be improved. There is therefore the need for measures to control the use of this chemical group, and the continuity of ANVISA to formally inform consumers (via website) since it stopped this activity in 2012.

Keywords: consumers, dithiocarbamates, apples, residues, PARA/ANVISA

\title{
Residuos de pesticidas del grupo ditiocarbamato en manzanas (Malus domestica Borkh.) in natura en Brasil - 10 años
}

\begin{abstract}
RESUMEN. Se realizó un análisis crítica de datos del Programa de Análisis de Residuos de Pesticidas (PARA) de la Agencia Nacional de Vigilancia Sanitaria (ANVISA) sobre los residuos de pesticidas del grupo químico ditiocarbamato (DTC) en manzanas (Malus mestica Borkh) comercializadas in natura durante diez años en Brasil. Fueron analizadas 1422 muestras de manzanas, de las cuales 605 tenían algún nivel de contaminación. El número de muestras positivas para el DTC fue del $32,1 \%$ en 2003 , ya el año siguiente (2004) se establece como el período con mayor número de muestras contaminadas, correspondiendo a 83,9\%, seguido del año de 2007 con el 81,9\%. Las manzanas alcanzaron niveles de 3,04 mg/kg en 2005, superando el límite máximo de residuos - LMR (2mg/kg) nacional. Independientemente de los datos reportados, el programa no informa cuántas y/o el porcentaje de muestras que supera el LMR, solamente fue posible observar que la presencia residual en las manzanas brasileñas, durante todo el período de evaluación fluctuaron en picos por encima del LMR en 2001/2002, 2005 2009, 2010 y 2012, exponiendo los consumidores a los efectos adversos de los DTCs. Teniendo en cuenta el rango de años de monitoreo realizada por parte del PARA y evaluados en este estudio, se observó que no hubo avances en los residuos detectados (reducción), mucho menos aumento en el número de muestras analizadas (oscilado en todos los períodos), así como en los laboratorios oficiales para análisis, lo que demuestra la necesidad de mejorar estos factores. Hay necesidad de que a ANVISA continúe informando oficialmente a los consumidores (a través de sitio web) ya que este proceso informativo se detuvo en 2012.
\end{abstract}

Palabras clave: Consumidores, ditiocarbamatos, manzanas, residuos PARA/ANVISA

\section{Introdução}

A agricultura está sempre em busca de ferramentas para combater doenças e pragas objetivando aumentar sua produtividade. O meio químico têm sido o mais utilizado, o que tem feito o Brasil ser o maior consumidor mundial de agrotóxicos desde 2008 (Londres, 2011, Braibante and Zappe, 2012). Sua aplicação em vários estágios de cultivo exerce papel fundamental na proteção e na preservação de alimentos, atuando como herbicidas, inseticidas, raticidas e fungicidas (Xavier and Spinosa, 2008, Collotta et al., 2013). Existem diversos grupos químicos de agrotóxicos, dentre eles os ditiocarbamatos (DTCs), cujos princípios ativos mais comumente utilizados permitidos no Brasil são o metiram, mancozebe, metam sódico, tiram, propinebe aplicados em diversos vegetais, incluindo maçãs (Malus domestica Borkh.) (Lemes, 2007) (Tabela 1)

A população está exposta aos efeitos maléficos pelo contato com esses produtos, seja através da ingestão de alimentos com seus resíduos ou, ainda, de forma indireta, pela contaminação ambiental (Bolognesi, 2003, Londres, 2011). O uso indiscriminado de agrotóxicos nos alimentos tem se tornado uma preocupação para o consumidor e as autoridades de saúde, em função da toxicidade que podem apresentar (Caldas and Souza, 2004, Londres, 2011). 
Tabela 1. Estruturas químicas de ditiocarbamatos de uso autorizado no Brasil

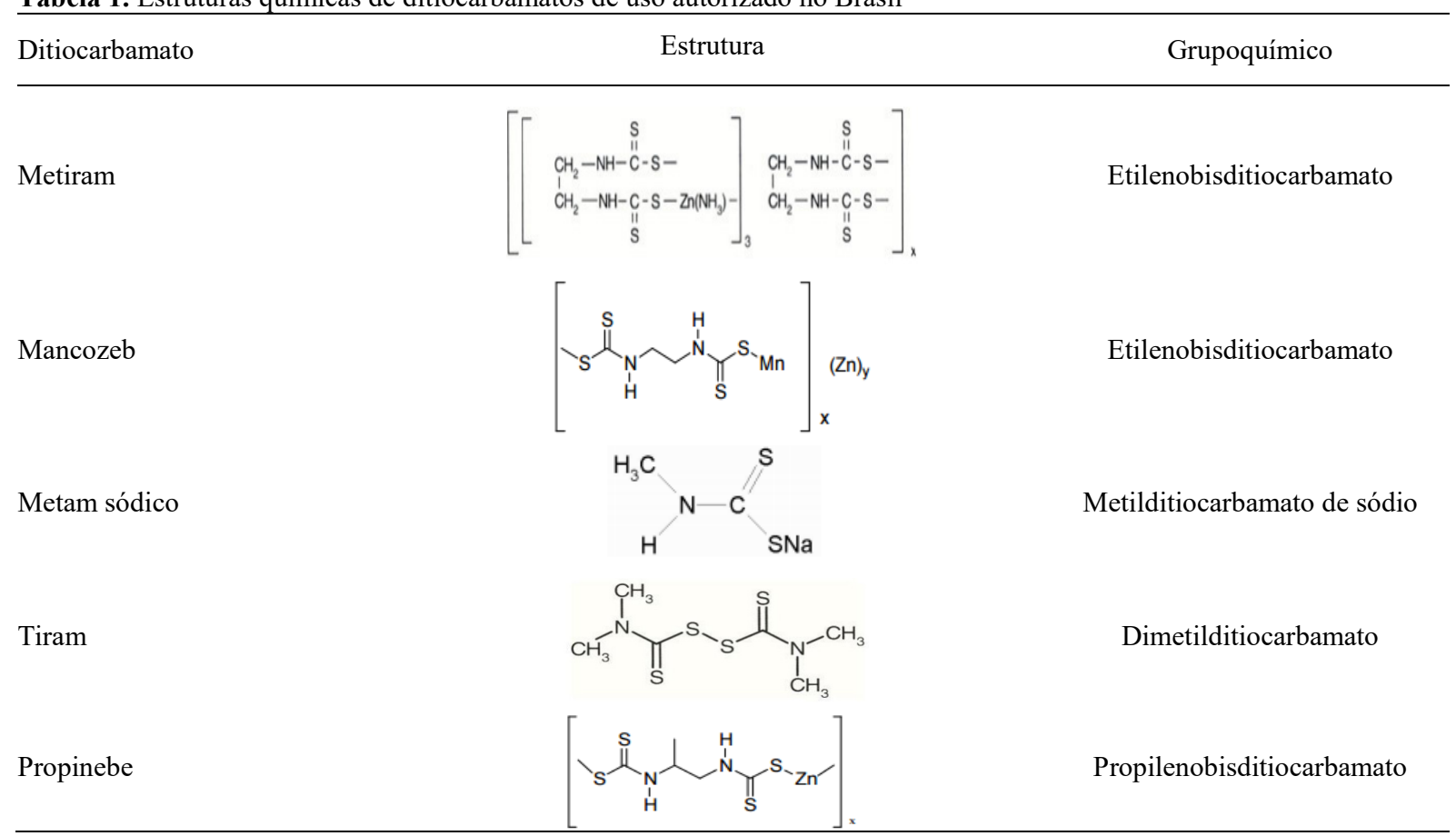

Fonte: Lemes (2007), Brasil (2012a).

No Brasil, a Agência Nacional de Vigilância Sanitária (ANVISA), é o órgão responsável em determinar os limites de resíduos desses produtos. Para tanto, foi criado em 2001 o Plano Nacional de Controle de Resíduos e Contaminantes (PARA), um programa federal que inspeciona e divulga dados do controle dos sistemas de produção e a respectiva qualidade e segurança dos produtos para consumo (Brasil, 2012).

A produção mundial de maçã vem crescendo anualmente, principalmente seu consumo in natura. Diversas doenças causadas por fungos (sarna da macieira, oídio, mancha de filosticta, mancha de marsonina, mancha necrótica foliar, bolor azul, fuligem e podridões amarga / carpelar / marrom) podem acometer as maçãs no campo e diferentes agrotóxicos são utilizados para controlar e/ou prevenir sua proliferação/infecção sendo o grupo principal o dos DTCs (Janisiewicz and Korsten, 2002, Scussel and Galvão, 2009). A presença de resíduos de DTCs em maçãs é um indicativo de que o agricultor não está utilizando esse agrotóxico de maneira adequada e/ou que deve dar maior atenção às boas práticas agrícolas (respeitando o período de carência e concentração recomendada de aplicação).

Considerando que o consumo de maçãs in natura (com casca) com resíduos de agrotóxicos associado às falhas no respeito ao período de carência, bem como de aplicação (altas concentrações / aplicações repetitivas) podem levar a intoxicação crônica em humanos, afetando, portanto a saúde pública (Scussel and Galvão, $\underline{2009)}$.

Este trabalho teve como objetivo realizar uma avaliação sobre a evolução na segurança das maçãs in natura comercializadas no Brasil (com ênfase nos resíduos de DTCs pela análise crítica dos dados reportados pelo programa PARA/ANVISA no período de 10 anos e exposição do consumidor).

\section{Material e Métodos}

Amostra: dados oficiais do PARA/ANVISA quanto a resíduos de agrotóxicos do Grupo químico DTCs obtidos de maçãs in natura (incluindo ambas as variedades, Gala e Fugi) comercializadas em diferentes estados brasileiros.

Instrumento de coleta de dados: a coleta dos dados se baseou na pesquisa de informações em um banco de dados do PARA/ANVISA. Este programa atua no Brasil desde 2001 avaliando a presença dos mais diversos resíduos de agrotóxicos em diferentes culturas.

Período da pesquisa: os dados da coleta corresponderam ao período de 2001 (ano de início do Programa) até 2012 (últimas informações registradas no PARA até a presente data). Total: 10 anos. Cabe reforçar que a ANVISA parou de fornecer / divulgar os dados a partir de 2013, portanto não disponíveis para consulta pública. 
Informações coletadas: no banco de dados do PARA foram coletadas as seguintes informações: (a) número de amostras analisadas / ano; (b) Laboratórios em que as amostras foram analisadas; (c) número de amostras positivas para resíduos de DTCs; (d) porcentagem de amostras contendo resíduos de DTCs; (e) níveis detectados; (f) intervalos de detecção das amostras / ano versus (g) LMR (limite máximo de resíduos) de DTCs estabelecido pela legislação brasileira.

Análise dos dados: após a coleta dessas informações, as mesmas foram reunidas em planilhas para posterior análise dos dados. O programa usado para a análise foi o Microsoft Excel, versão 2010. A partir das informações obtidas, essas foram distribuídas em diferentes gráficos para melhor interpretação dos resultados (abordando os diversos aspectos do presente estudo). Nesta perspectiva, as informações foram correlacionadas nos seguintes aspectos: (a) levantamento do número de amostras analisadas pela própria ANVISA versus amostras realizadas por laboratórios parceiros/oficiais; comparação da produção anual brasileira de maçã versus número de amostras coletadas e analisadas pelo PARA/ANVISA por ano; (c) comparação entre número de amostra analisadas versus amostras com detecção de resíduos; (d) avaliação dos níveis de resíduos máximos e os mínimos dos intervalos de detecção e (e) por fim, os dados obtidos foram comparados com outros já descritos na literatura.

Especificações: (a) variedade de maçãs: a ANVISA não específica nos dados liberados, se são provenientes das variedades Fugi, Gala ou outras; (b)coleta das amostras: é realizada por estado, pelos órgãos oficiais locais;(c) local: as amostras são coletadas em mercados varejistas, local onde a população adquire os alimentos; (d)Laboratórios: as amostras são enviadas a laboratórios parceiros da ANVISA para análise; (e) princípios ativos do Grupo dos DTCs envolvidos: metiram, mancozeb, metam sódico, tiram, propineb dentre outros; (f) análises: são realizadas principalmente por cromatografia gasosa com detecção de massa(s), sendo que os métodos são validados pelo próprio laboratório responsável pelas análises, bem como pelo INMETRO e pouco são os dados reportados que vêm acompanhados de seus LOD e LOQ; (g) dados: são liberados como resíduos do grupo dos DTCs (não são especificados os princípios ativos presentes em cada amostra/ resíduo detectado); (g.1) níveis: são liberados em faixas (não individualmente). (g.2) início: como o PARA iniciou em 2001 - os primeiros dados de resíduos obtidos foram liberados para dois anos (2001 \& 2002); (g.3) lacunas: não foram registrado pelo PARA, dados do total de número de amostra positivas em 2008/2009/2012, embora sim, para o número dos níveis detectados.

\section{Resultados e discussão}

A partir dos dados coletados, foi possível obter uma perspectiva da contaminação de maçãs produzidas no Brasil, quanto a resíduos de DTCs e sua distribuição ao longo do período de estudo (10 anos). As Figuras de 1 a 4 apresentam os dados registrados pelo PARA/ANVISA quanto ao número e identificação dos laboratórios oficiais responsáveis pela realização das análises, bem como a relação entre amostras e suas variáveis (número de positivas/níveis/faixa), produção de maçã versus número de amostras analisadas/ano e não conformidades detectadas quanto ao LMR (2 $\mathrm{mg} / \mathrm{kg}$ ) estabelecido pela legislação vigente.

\section{Amostras analisadas e laboratórios oficiais parceiros}

Laboratórios parceiros: do total de 1422 amostras de maçãs comercializadas em vários estados do país e que foram submetidas a análises (2001 a 2012) de resíduos de DTCs, 738 amostras foram avaliadas por laboratórios oficiais parceiros do programa PARA enquanto lotes de 634 pela própria ANVISA e é de sua responsabilidade (não informando a procedência do laboratório). Os laboratórios que fazem parte e considerados parceiros para realizar e divulgar dados oficiais do PARA foram: a Fundação Ezequiel Dias (FUNED) em Minas Gerais, o Instituto Adolfo Lutz (IAL) em São Paulo e o Laboratório Central de Saúde Pública (LACEN - PR) no Paraná (Figura 1).

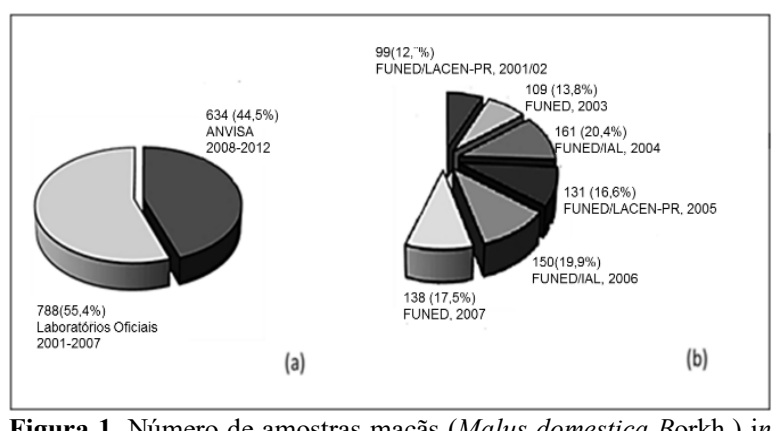

Figura 1. Número de amostras maçãs (Malus domestica Borkh.) in natura analisadas pela Agência Nacional de Vigilância Sanitária e pelos laboratórios oficiais parceiros do Programa Nacional de Resíduos de Agrotóxicos (a) total (b) laboratório/ano. 
Cabe salientar que poucos são os laboratórios no Brasil credenciados para realizar análises de resíduos de agrotóxicos. Além dos citados acima, outros que podem participar são a Fundação de Ciência e Tecnologia (CIENTEC) no Rio Grande do Sul, Instituto Biológico (IB) em São Paulo e Instituto de Tecnologia (ITEP) em Pernambuco. Santa Catarina é o único estado da região Sul e Sudeste que não possui laboratório oficial para realizar análise de rotina para agrotóxicos, precisando enviar amostras para outros estados, embora seja o maior produtor nacional de maçã (Scussel and Galvão, 2009).

\section{Resíduos de ditiocarbamatos detectados}

Maçãs analisadas / ano e detecção de DTCs (2001 a 2012): em relação às amostras analisadas, foi possível perceber (em praticamente todas as safras de maçãs), um número significativo apresentando resíduos de DTCs (Figura 2).Do total avaliado (1422 amostras) durante os anos de monitoramento, 605 amostras apresentaram resultados insatisfatórios. Cabe salientar que, nos anos correspondentes a 2008, 2009 e 2012 foi divulgado apenas o número de amostras analisadas, entretanto a ANVISA não informou o número de amostras que estavam contaminadas com os resíduos de DTCs. Constam apenas os intervalos dos valores detectados, fato que dificultou parte da avaliação, já que foram os anos com maior número de amostras analisadas, principalmente 2008, $2009 \quad(240$ e 170, respectivamente). De todo o período de estudo, o ano de 2004 se destacou por apresentar o maior número de amostras com resíduos de DTCs. Naquele ano, foram avaliadas 161 amostras, dessas, em 135 foram encontrados níveis de DTCs, equivalendo a $83,9 \%$ do total. Já no ano de 2007, foi analisado um número menor de amostras (138), em que por sua vez, 113 apresentaram resultados positivos para DTCs, correspondendo a $81,9 \%$. No outro extremo, o ano de 2003 apontou os menores resultados, embora com 109 amostras analisadas, somente $35 \quad(32,1 \%)$ exibiram irregularidades. Apesar de várias amostras de maçãs apresentarem valores acima do permitido, não foi possível saber com exatidão o número dessas, pois o Programa divulga apenas os intervalos de detecção. A Figura 2 apresenta a relação entre o número de amostras de maçãs analisadas pela ANVISA anualmente e o número de maçãs que apresentaram resíduos de agrotóxicos.
O PARA, tem diagnosticado desde 2001 (início de suas atividades) níveis de agrotóxicos em diversas culturas, muitos desses, acima do permitido pela legislação, por isso o mesmo vem sendo considerado atualmente como a principal ferramenta indicativa do uso irregular de agrotóxicos em alimentos no Brasil, assumindo, portanto, um papel significativo na garantia da segurança alimentar. Até 2012 o Programa validou metodologia para analisar 22 culturas, sendo elas: arroz, abobrinha, abacaxi, alface, banana, batata, beterraba, cebola, cenoura, couve, feijão, laranja, maçã, mamão, manga, milho, morango, pepino, pimentão, repolho, tomate e uva, atuando nas 27 Unidades Federativas (Brasil, 2012). Contudo, o PARA ainda necessita ser aprimorado quanto à divulgação dos dados a população já que parou de divulgar a partir de 2013.

A Agência de Proteção Ambiental (EPA) dos Estados Unidos reportou que DTCs têm sido detectados em seu país, com níveis acima dos LMR, em várias culturas incluindo maçãs, morangos, tomates, pêras e pimentões (EPA, 2009). Essa informação vai de encontro ao presente trabalho, pois foi observada a presença desse resíduo em grande parte das amostras (em níveis variados). Em 2012, o PARA constatou que o maior número de irregularidades em alimentos envolvia DTCs, seguido de outros princípios ativos, tais como carbendazim, clorpirifos, acefato e piraclostrobina. Especificamente na maçã, além de DTCs, vem sendo registrado níveis acima do tolerado de acefato, carbosulfano, fenitrotiona, lambada-cialotrina, metidationa, permetrina, protiofos e tebuconazol.

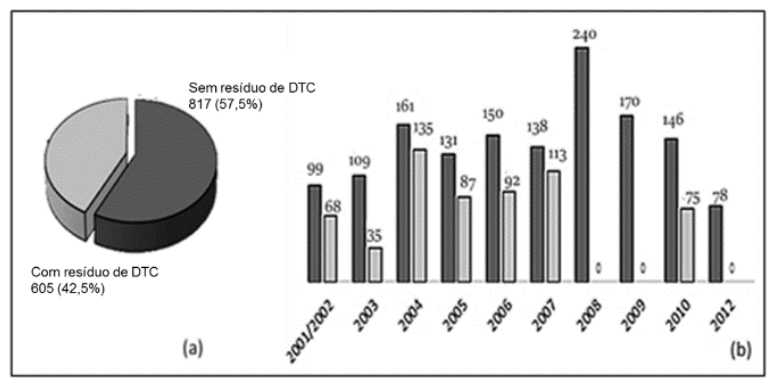

Figura 2. Relação entre o número de amostras de maçãs (Malus domestica Borkh.) avaliadas e as que apresentaram resíduos de ditiocarbamtos (a) todo o período (2001-2012) e (b) por ano.

Resíduos versus consumo in natura elou com casca: a preocupação com a presença desses resíduos, detectados muitas vezes, acima do tolerado, principalmente em vegetais, pode ser explicada pelo fato de que em sua grande maioria, 
são consumidos in natura. A maçã é um exemplo, pois cerca de $80 \%$ dela é assim consumida, muitas vezes inclusive sem a higienização correta, expondo o consumidor aos efeitos nocivos dos agrotóxicos. Somado ao exposto, os efeitos cumulativos à saúde pela ingestão de maçãs e outros alimentos contaminados por DTCs são pouco conhecidos. Mesmo estando dentro do LMR, sabe-se que os DTCs possuem grande potencial toxicológico, principalmente pelo seu uso intenso e constante contra doenças das maçãs (Jardim et al., 2009). Vale destacar que o uso de agrotóxicos é muito corriqueiro na horticultura, pois é a forma mais comum encontrada para o controle de doenças e pragas. Todavia, os resíduos dessas substâncias na superfície de frutos nem sempre são removidos. Para que isso ocorra depende de algumas características, como natureza da fruta, bem como o tipo e quantidade de agrotóxico usado durante a pulverização e fatores de degradação (luz- calor/UV; aroxidação; umidade-hidrólise) (Scussel and Galvão, 2009, Londres, 2011, Zhang et al., 2015).

Resistência de pragas: um dos motivos, que têm levado a um considerável aumento dessas substâncias na maçã, decorre do aumento de resistência das pragas. O que exige a aplicação de doses mais expressivas de agrotóxicos, consequentemente intensificando esse problema de saúde pública (Jardim et al., 2009). Outro fator associado com a detecção de níveis de resíduos acima do permitido em algumas safras, pode estar relacionado com mudanças climáticas extremas, o que facilita o desenvolvimento de pragas e consequentemente o uso mais intenso de agrotóxicos como os DTCs.

Produção de maçãs $\mathrm{x}$ quantidade efetiva de amostras analisadas/ano: contudo, ao considerar a produção anual brasileira de maçãs (Figura 3), que oscilou no período de estudo de 600.000 a quase 1.400 .000 toneladas, é possível perceber que o número de amostras analisadas por ano pelo PARA/ANVISA, ainda é reduzido e não representativo de toda a produção. Isso pode ser explicado pela reduzida quantidade de laboratórios oficiais que realizam esse tipo de análise, e que necessita ser ampliada.

\section{Limite máximo de resíduo vs. níveis detectados em} maças

LMR: de acordo com o Decreto ${ }^{\circ} 4.074$, de 4 de janeiro de 2002, LMR é definido como a quantidade máxima de resíduo de agrotóxico oficialmente aceita no alimento, em decorrência da aplicação adequada numa fase específica, desde sua produção até o consumo, expresso em $\mathrm{mg} / \mathrm{kg}$ (Brasil, 2002). Seguindo esta definição, é possível perceber que a determinação de um LMR para cada produto é uma forma de garantir a saúde e segurança do consumidor. Para o grupo de compostos químicos (DTCs), com efeito fungicida/ inseticida, o LMR em maçãs estabelecido pelo Ministério da Saúde, através de sua Agência fiscalizadora (a ANVISA) é de 2 $\mathrm{mg} / \mathrm{kg}$ (Brasil, 2012).

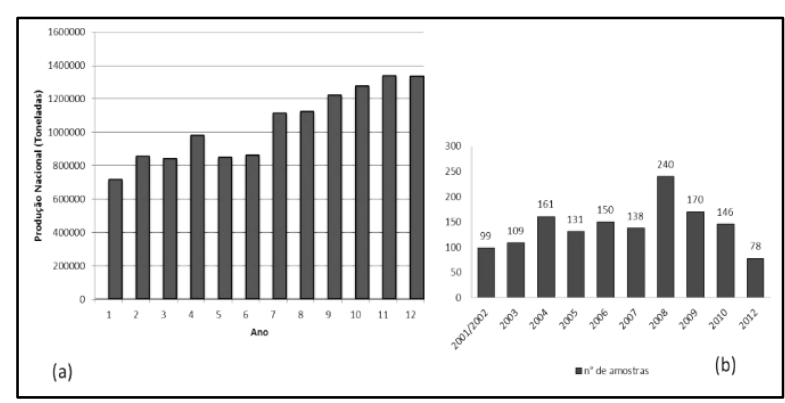

Figura 3. Maçãs (Malus domestica Borkh.) no Brasil período de 2001 a 2012: (a) produção anual (toneladas) versus (b) número total de amostras analisadas pelo PARA/ANVISA

LMR versus níveis detectados: no presente estudo, foi possível observar que das faixas (denominadas de intervalos de detecção pelo PARA) reportadas para essa fruta, os níveis máximos não ultrapassaram $3,04 \mathrm{mg} / \mathrm{kg}$, o qual foi o nível mais elevado em 2005 (ou seja, $1,04 \mathrm{mg} / \mathrm{kg}$ acima do LMR) (Figura 4). Em 2010, amostras atingiram resíduo máximo de $2,76 \mathrm{mg} / \mathrm{Kg}$. Os níveis também ultrapassaram o LMR em 2001/02, 2009 e 2012 com valores máximos de até 2,10, 2,24 e $2,53 \mathrm{mg} / \mathrm{kg}$, respectivamente. Por outro lado, as amostras apresentaram-se satisfatórias nos períodos de 2003, 2004, 2006 e 2007 com intervalos de detecção baixos $(0,08-1,48 ; 0,20$ 2,$00 ; \quad 0,08-1,63 \quad$ e $\quad<$ LOQ- $1,3 \mathrm{mg} / \mathrm{kg}$, respectivamente) em um número considerável de amostras: 32,$1 ; 83,9 ; 61,3$ e $81,9 \%$.

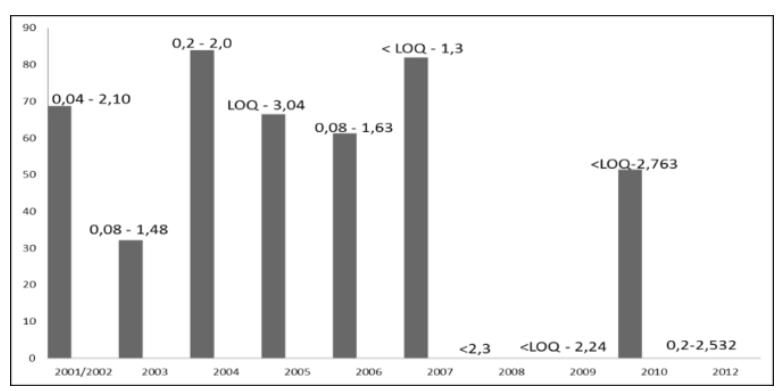

Figura 4. Porcentagem de amostras de maçãs (Malus domestica Borkh.) detectadas com resíduos de ditiocarbamatos e os intervalos de detecção $(\mathrm{mg} / \mathrm{kg})$ 
Clareza: cabe salientar que os dados, quando as amostras se apresentavam em níveis acima do LMR, não especificavam o número $\mathrm{e} a / \mathrm{ou}$ porcentagem delas que efetivamente ultrapassaram esse limite, dificultando saber se foi um número reduzido ou elevado que o ultrapassou. Outro detalhe é quanto aos dados da validação do método utilizado pelos laboratórios, poucos foram os anos que os dados de DTCs registrados eram acompanhados dos seus respectivos LODs e LOQs.

Vários estudos têm demonstrado à presença de resíduos de agrotóxicos dos mais diversos grupos químicos (incluindo os DTCs) em alimentos, principalmente de origem vegetal, denotando a relevância dos programas de análise de resíduos.

Gorenstein (2000) analisou 1.439 amostras de produtos hortifrutícolas (morango, tomate, mamão, pêssego e uva), quanto à presença de diferentes princípios ativos (endosufan, cloratalonil, dimetoato, metamidofós, procimidone, vinclozolina, acefato e folpe). $\mathrm{O}$ autor relatou que $69,4 \%$ das amostras estavam sem resíduos de agrotóxicos (ou seja: <LOQ), 15,2\% com presença de resíduos em quantidades $<$ LMR e somente $1,4 \%$ das amostras apresentaram resíduos de compostos não permitidos para a cultura (>LMR). Um estudo realizado por Lopes and Dórea (2003) avaliou amostras de feijão comercializadas em Aracaju-Sergipe quanto à contaminação por vários princípios ativos (diazinon, propanil, malation, cipermetrina e deltametrina). Os resultados mostraram concentrações acima do LMR, dos agrotóxicos diazion, propanil e deltametrina, com valores médios de $0,64, \quad 0,39$ e $1,83 \mathrm{mg} / \mathrm{kg}$, respectivamente.

Caldas and Souza (2004) avaliaram resíduos de DTCs em 520 amostras de frutas (mamão, banana, maçã, morango, laranja, tomate) bem como outros vegetais (batata, arroz e feijão). Desses, o mamão, a banana e a maçã foram os vegetais mais contaminados, com 100,75 e $65 \%$ de resíduos (>LOQ: $0,10 \mathrm{mg} / \mathrm{kg}$ ), respectivamente. Os autores relataram que maiores níveis de resíduos foram encontrados em morango $(3,8 \mathrm{mg} / \mathrm{kg})$, banana e tomate $(3,3 \mathrm{mg} / \mathrm{kg})$.

Esses estudos comprovam a suscetibilidade dos consumidores quanto à exposição diária aos diversos princípios ativos, através da alimentação, que é considerada uma das principais vias de exposição.

\section{Conclusões}

Um número significativo de amostras de maçãs analisadas no período de estudo (2001 a 2012) apresentou algum resíduo de DTCs, contudo, a maioria estava com níveis dentro e/ou no limiar do LMR permitido pela legislação. O ano de 2004, embora representado como o de maior número de amostras analisadas, bem como de positivas, os níveis $(0,1-2,0 \mathrm{mg} / \mathrm{kg})$ estavam dentro do LMR permitido, sendo as análises realizadas pelo laboratório de São Paulo (IAL). Em contrapartida, o ano de 2003 foi o que teve menor número de amostras e níveis detectados ( $<\mathrm{LMR}$ ) desse grupo químico, sendo as análises efetuadas pelo laboratório parceiro de Minas Gerais (FUNED).

A partir dos dados obtidos, foi possui concluir a importância da atuação do PARA no monitoramento de resíduos de agrotóxicos (especificamente de DTCs) em maçãs, já que a mesma é muito consumida e necessita ser monitorada.

Considerando a faixa de anos do monitoramento realizado pelo PARA, foi possível observar que não houve evolução nos resíduos detectados (redução), muito menos acréscimo no número de amostras analisadas (oscilou em todos os períodos), bem como de laboratório oficiais para análise (somente 4 participaram). Urge, o Programa reportar / deixar mais claro o número e porcentagem de amostras efetivamente acima do LMR, em vez de somente expressar em faixas (inclui tanto os $<$ quanto $>$ do LMR) o que leva a erro de interpretação pelo consumidor.

Os dados obtidos também evidenciam a importância e necessidade da adoção das boas práticas agrícolas nas diversas etapas de produção da fruta, bem como a necessidade de respeitar o período de carência dos produtos e seguir as informações presentes no receituário agronômico, para posterior comercialização segura dos produtos cultivados e também consumo pela própria família rural livre de agrotóxicos. Há, portanto necessidade da implementação e continuidade da ANVISA de informar oficialmente (via site), uma vez que essa atividade parou em 2012 bem como, de disponibilidade de um número maior de laboratórios oficias parceiros para a realização das análises, inclusive no estado de maior produção da fruta, Santa Catarina. 


\section{Referências Bibliográficas}

Bolognesi, C. 2003. Genotoxicity of pesticides: a review of human biomonitoring studies. Mutation Research, 543, 251-272.

Braibante, M. E. F. \& Zappe, J. A. 2012. A química dos agrotóxicos. Química Nova na Escola, 34, 10-15.

Brasil. Decreto $\mathrm{n}^{\circ} 4.074$, de 4 de janeiro de 2002.Regulamenta a Lei $\mathrm{n}^{0} 7.802$, de 11 de julho de 1989, que dispõe sobre a pesquisa, a experimentação, a produção, a embalagem e rotulagem, o transporte, o armazenamento, a comercialização, a propaganda comercial, a utilização, a importação, a exportação, o destino final dos resíduos e embalagens, o registro, a classificação, o controle, a inspeção e a fiscalização de agrotóxicos, seus componentes e afins, e dá outras providências. Diário Oficial da República Federativa do Brasil, Poder Executivo, Brasília, DF, 4 jan. 2002.

Brasil. Programa de Análise de Resíduos de Agrotóxicos em Alimentos - PARA. Relatório complementar relativo à segunda etapa das análises de amostras coletadas em 2012. 2012. Disponívelem: $<$ http://portal.anvisa.gov.br/doc uments/111215/117818/Relat $\% 25 \mathrm{C} 3 \% 25 \mathrm{~B} 3 \mathrm{ri}$ o\%2BPARA $\% 2 B 2012 \% 2 B 2 \% 25 \mathrm{C} 2 \% 25 \mathrm{AA}$ $\% 2 B$ Etapa\%2B-\%2B17 10 14Final.pdf/3bc220f9-8475-44ad-9d96cbbc988e28fa>. Acesso em: 23 de maio 2016.

Caldas, E. D. \& Souza, L. C. K. R. 2004. Chronic dietary risk for pesticide residues in food in Brazil: an update. Food Additives and Contaminants, 21, 1057-1064.

Collotta, M., Bertazzi, P. A. \& Bollati, V. 2013. Epigenetics and pesticides. Toxicology, 307, 35-41.

EPA - Environmental Protection Agency. Ethylenethiourea - identification, toxicity, use, water pollution potential, ecological, toxicity and regulatory information. 2009. Disponível em:

$<$ http://www.pesticideinfo.org/Detail_Chemic al.jsp?Rec_Id=PC33208 $>$. Acesso em: 1 de junho $201 \overline{6}$.

Gorenstein, O. 2000. Uma abordagem sobre resíduos de agrotóxicos em alimentos frescos. Informações Econômicas, São Paulo, 1, 1-10.
Janisiewicz, W. J. \& Korsten, L. 2002. Biological control of postharvest diseases of fruits. Annual Review of Phytopathology, 40, 411441.

Jardim, I. C. S. F., Andrade, J. A. \& Queiroz, S. C. N. 2009. Resíduos de agrotóxicos em alimentos: uma preocupação ambiental globalUm enfoque às maçãs. Química Nova, 332, 996-1012.

Lemes, V. R. R. 2007. Avaliação de resíduos de etilenotiouréia (ETU) em frutas comercializadas na cidade de São Paulo. Universidade de São Paulo, São Paulo.

Londres, F. 2011. Agrotóxicos no Brasil: um guia para ação em defesa da vida. Assessoria e Serviços a Projetos em Agricultura Alternativa, Rio de Janeiro.

Lopes, W. G. \& Dórea, H. S. 2003. Determinação de pesticidas em feijão por dispersão da matriz em fase sólida (DMFS). Revista de Ecotoxicologia e Meio Ambiente, 13, 73-82.

Scussel, V. M. \& Galvão, S. 2009. Contaminação de maçãs e seus derivados por agrotóxicos e micotoxinas. In: Stadnik, M. J. (ed.) Avanços genéticos da macieira no controle de doenças. Universidade Federal de Santa Catarina, Florianópolis, Santa Catarina.

Xavier, F. G. \& Spinosa, H. S. 2008. Toxicologia dos praguicidas. In: Spinosa, H. S., Górniak, S. L. \& Neto, J. P. (eds.) Toxicologia aplicada à medicina veterinária. Manole, Barueri.

Zhang, M., Zeiss, M. R. \& Geng, S. 2015. Agricultural pesticide use and food safety: California's model. Journal of Integrative Agriculture, 14, 2340-2357.

\author{
Article History: \\ Received 11 November 2016 \\ Accepted 17 January 2017 \\ Available on line 27 March 2017
}

License information: This is an open-access article distributed under the terms of the Creative Commons Attribution License 4.0, which permits unrestricted use, distribution, and reproduction in any medium, provided the original work is properly cited. 